\title{
Malgacheliodes guillaumeti n. gen., n. sp. (Acari, Oribatida, Pheroliodidae) de Madagascar
}

\author{
Nestor FERNANDEZ \\ Consejo Nacional de Investigaciones Científicas y Técnicas (CONICET), \\ Universidad Nacional de La Pampa, \\ Facultad de Ciencias Exactas y Naturales, \\ Uruguay 151, (6300) Santa Rosa, La Pampa (Argentina) \\ nesfernan@yahoo.fr \\ Régis CLEVA \\ Muséum national d'Histoire naturelle, Direction des collections, \\ case postale 53, 57 rue Cuvier, F-75231 Paris cedex 05 (France) \\ cleva@mnhn.fr
}

MOTS CLÉS

Acari,

Oribatida,

Pheroliodidae,

Madagascar,

genre nouveau,

espèce nouvelle.
Fernandez N. \& Cleva R. 2010. - Malgacheliodes guillaumeti n. gen., n. sp. (Acari, Oribatida, Pheroliodidae) de Madagascar. Zoosystema 32 (4): 567-583.

\section{RÉSUMÉ}

Une série de spécimens d'oribates de la famille Pheroliodidae appartenant à un nouveau genre et une nouvelle espèce, Malgacheliodes guillaumeti n. gen., n. sp. a été récoltée à Madagascar dans le massif montagneux de l'Andringitra, situé dans le tiers sud de l'île. Les stases adulte et immatures sont décrites et illustrées, une clé des genres de la famille est proposée. Malgacheliodes n. gen., proche de Pheroliodes, se différencie de ce dernier par un ensemble de caractères qui justifient l'établissement d'un nouveau genre: 8 paires de poils génitaux disposés sur deux rangs; dent pédotectale, pointe custodiale et discidium présents; trachée I vestigiale; apophyse antilatérodorsale du tibia I avec 2 cuvettes; présence du poil compagnon $d$. La nouvelle espèce se caractérise par, chez les adultes: sensillus en massue aplatie; i $p$ en forme de demi-cercle; bordures épimériques ne correspondant pas exactement avec les apodèmes respectifs; demi-apodèmes 2 et $s j$ terminant loin du plan de symétrie; fémurs trachéens, carénés. Et chez les immatures: trachée I présente; crispins complets: bien développés sur tarses, tibias et génuals dès la stase larvaire; crispin du fémur IV bien visible à partir de la deutonymphe; $\varphi_{1}$ et $\varphi_{2}$ placés dès la deutonymphe dans deux cuvettes séparées. 


\begin{abstract}
Malgacheliodes guillaumeti $n$. gen., n. sp. (Acari, Oribatida, Pheroliodidae) from Madagascar.

A series of oribatid specimens of the family Pheroliodidae belonging to a new genus and new species, Malgacheliodes guillaumeti n. gen., n. sp. have been collected in Madagascar, in the Andringitra mountain, located in the southern third of the island. The adult and immature stasis are described and illustrated and a key of the family genera is proposed. Malgacheliodes n. gen. is close to Pheroliodes, but can be separated from the latter by a set of characters that justify the establishment of a new genus: eight pairs of genital setae, arranged in two rows; pedotectal tooth, custodial point and discidium present; trachea 1 vestigial; distal apophyse of tibia I with two hollows; seta $d$ present. The new species is characterized by, in the adults: sensillus, a flattened club; ip half-circle shaped; epimeric borders not exactly

KEY WORDS

Acari, Oribatida,

Pheroliodidae, Madagascar, new genus, new species.

corresponding with respective apodemes; half apodemes 2 and $s j$ ending far from the plan of symetry; femora carenated, with tracheas. And in the immatures: trachea I present; sockets complete, well developped on tarsus, tibias and genu, already present on the larva; socket of femur IV visible from deutonymphal stase; $\varphi_{1}$ and $\varphi_{2}$ housed in two separated hollows from deutonymphal stase.
\end{abstract}

\section{INTRODUCTION}

La famille Pheroliodidae a été établie par Paschoal (1987b) avec quatre genres: Licnoliodes Grandjean, 1931 (espèce type Licnoliodes andrei Grandjean, 1931); Octoliodes Paschoal, 1987 (espèce type Pedrocortesia luteomarginata Hammer, 1966); Pheroliodes Grandjean, 1964 (espèce type Cymberemaeus wehnckei Willmann, 1930) et Lopholiodes Paschoal, 1987 (espèce type Lopholiodes micropunctinatum Paschoal, 1987). Les deux espèces de Plateremaeoidea Trägårdh, 1931 de Madagascar, décrites par Balogh en 1962, Plateremaeus legendrei et Plateremaeus glaber, ont ensuite été étudiées par Paschoal (1987a, 1989b), qui a créé les nouveaux genres Paralopheremaeus Paschoal, 1987 pour la première de ces espèces, placé dans la famille Plateremaeidae Trägårdh, 1931 et Nooliodes Paschoal, 1989 pour la seconde, ce dernier devenant le genre type de la nouvelle famille Nooliodidae Paschoal, 1989.
La même année Paschoal (1989b) créa la famille Lyrifissellidae, avec le genre Lyrifissella Paschoal 1989 et l'espèce type Pedrocortesella latoclava Hammer, 1966.

En 1992, Balogh \& Balogh reconnaissent la famille Pheroliodidae composée des quatre genres, comme l'avait proposé Paschoal en 1987, ainsi que la famille Nooliodidae, dans laquelle ils placent les genres Flammeremaeus Balogh, 1968 (espèce type F. gressiti Balogh, 1968) et Nooliodes.

Mahunka (1997) cite Nooliodes, qu'il place dans la famille Plateremaeidae, et en 2002, citant les deux espèces de Madagascar, considère les genres Nooliodes et Paralopheremaeus comme faisant partie des Plateremaeidae.

Subias (2004, actualisé 2009) considère la famille Pheroliodidae, constituée par les genres Lopholiodes, Lyrifissella, Nooliodes et Pheroliodes. Il place le genre Paralopheremaeus dans la famille Plateremaeidae, et ne reconnait pas le genre Octoliodes qu'il considère synonyme de Lopholiodes; il ne mentionne pas 
les Nooliodidae ni les Lyriffissellidae. Dans cette situation extrêmement confuse, nous suivons provisoirement la position de Subias.

Nous avons placé le nouveau genre Malgacheliodes dans les Pheroliodidae compte tenu des caractères suivants : adulte avec scalps; cuticule du notogaster à concavités arrondies; $\mathrm{Ng}: 5 ; \mathrm{G}: 8$, en deux rangs (5 et 3); trachées I, sj et III présentes; apophyse pédotectale, pointe custodiale et discidium présents; crispins complets aux génuaux, tibias et tarses, partiels aux fémurs; apophyse du tibia I en deux cuvettes séparées, poil compagnon $d$ présent; formule gastronotique (7-6-5), génitale (1-4-7-8), aggénitale (0-1-1-1).

Des trois genres crées par Paschoal (Lopholiodes, Lyrifissiella et Nooliodes), celui qui nous pose le plus de problèmes est le genre Nooliodes, établi à partir de l'espèce Plateremaeus glaber, de Madagascar.

Plateremaeus glaber a été décrite de façon très sommaire et illustrée seulement par deux petites figures de l'animal entier, une vue dorsale et une vue ventrale (Balogh 1962: 420, figs 5; 6). Quand Paschoal, en 1989, redécrit l'espèce sous la nouvelle combinaison Nooliodes glaber, il indique (Paschoal 1989a: 179) «The type species, Plateremaeus glaber Balogh, from Madagascar, is redescribed based on previous description and figures. The new genus is close to Octoliodes Paschoal, 1987 family Pheroliodidae Paschoal. ", et un peu plus loin: "Nooliodes glaber (Balogh) comb. n. Plateremaeus glaber Balogh, 1962: 421, figs 7; 8. Types - Holotype plus nine paratypes collected in Madagascar; substrate and collecting date not mentioned: Depositary: Possibly at the "Collection of the Zoology Department", "Hungarian Natural History Museum", Budapest, Hungary». Page 181, Paschoal indique encore: "Description - (Based on the description and figures provided by Balogh, 1962). » Paschoal n'a donc pas réexaminé le matériel type. Il fait pourtant une redescription de $P$. glaber où il décrit une série de caractères qu'on ne voit pas sur les figures de Balogh, pas plus qu'ils ne sont mentionnés dans le texte et qui seront, malgré cela, utilisés pour établir le nouveau genre Nooliodes! Nous ne tiendrons compte ici que des seuls caractères qui figurent dans le travail des deux auteurs.
Une étude de l'espèce type du genre permettrait peut-être d'éclaircir le problème.

Nous considérons donc finalement que les Pheroliodidae comportent cinq genres: Pheroliodes, Nooliodes, Lopholiodes, Lyrifissella et Malgacheliodes n. gen., le plus proche de Malgacheliodes n. gen. étant Pheroliodes.

\section{MATÉRIEL ET MÉTHODE}

Le matériel étudié dans cette note a été récolté dans le cadre du Programme no 225 (RCP 225) mis en place par le CNRS entre 1970 et 1973 pour l'étude des écosystèmes montagnards de Madagascar (Paulian 1961; Paulian et al. 1971, 1973).

Les animaux ont été extraits de la litière par voie sèche (Berlese-Tullgren) et récoltés dans l'alcool à $70^{\circ}$.

La méthodologie utilisée pour les observations en microscopie optique et au microscope électronique à balayage (MEB) est la même que dans les travaux précédents (voir par exemple Fernandez \& Cleva 2010).

\section{ÉTUDE DES APODÈMES}

Il faut laisser séjourner les animaux longuement dans l'acide lactique chaud (de 1 journée à plusieurs semaines), enlever le notogaster, couper les animaux longitudinalement en restant assez loin du plan de symétrie, et faire l'observation de l'intérieur, latéralement et postérieurement, à cause des épaississements cuticulaires internes qui empêchent d'étudier les apodèmes en les observant de l'extérieur.

\section{ÉTUDE DU SYSTÈME TRACHÉEN}

Plusieurs exemplaires sont nécessaires; dissection obligatoire; animaux coupés longitudinalement; éclaircissement progressif dans l'acide lactique et coloration au noir de chlorazol; observations de la paroi cotyloïde de l'intérieur de l'animal, en suivant la progression de la coloration.

\section{ÉTUDES DES STASES IMMATURES}

La description sera faite sur la base de celle de Pheroliodes wehnckei (Willmann, 1930) (Grandjean 1964); nous ne mentionnerons que les différences observées avec l'espèce indiquée. 


\section{ABRÉVIATIONS}

Les abréviations indiquées dans les figures sont celles de la terminologie utilisée par Grandjean (Travé \& Vachon 1975), avec en plus:

1 scalp de la protonymphe;

2 scalp de la deutonymphe;

3 scalp de la tritonymphe;

b.ob branches obliques;

b.p branches ramifiées;

c.a concavités arrondies;

c.b couche basale;

c.g colonnes grandes;

c.m.l colonnes moyennes;

c.p colonnes petites;

c.p.ch petit choux-fleur;

e.a.r épaississements antérieurs ramifiés;

e.c.o épaississements cuticulaires;

L scalp larvaire;

p.cus pointe custodiale;

p.p petites boules;

s.t.a sillon transversal antérieur;

z.c zone centrale;

z.dep zone déprimée;

z.e zone élevée.

Le matériel type est déposé au Muséum national d'Histoire naturelle, Paris (MNHN).

Les photos au MEB ont été réalisées au Service commun de Microscopie électronique du MNHN.

\section{SYSTÉMATIQUE}

Famille PHeroliodidAe Paschoal, 1987

Genre Malgacheliodes n. gen.

ESPÈCE TYPE. - Malgacheliodes guillaumeti n. sp. par présente désignation.

ÉTYMOLOGIE. - Contraction de l'origine géographique et de l'appartenance à la famille Pheroliodidae.

DiAgnose. - Euphérédermes. Adulte avec scalps. Cérotégument à colonnes de différentes tailles. Cuticule du notogaster à concavités arrondies. Insertion de ro à l'arrière de le; ea présente. Capuchon rostral avec visière. $\mathrm{Ng}: 5$. G: 8 , en deux rangs ( 5 et 3 ); ag situé près du volet génital, vers l'arrière. Trachées I, $s j$ et III présentes, I très régressive. Chaetotaxie épimérique (3-1-3-3); au niveau $4 a, 4 b$ parfois une faible néotrichie. Apophyse pédotectale, pointe custodiale et discidium présents. Acetabulum IV placé très haut, à la limite du bouclier ventral. Crispins complets aux génuaux, tibias et tarses, partiels aux fémurs.
Apophyse du tibia I en deux cuvettes séparées, logeant $\varphi_{1}$ et le poil compagnon $d$ et $\varphi_{2}$ respectivement. Formule gastronotique (7-6-5), génitale (1-4-7-8), aggénitale (0-1$1-1)$, coxisternales $(3-1-2)(3-1-2-1)(3-1-3-2)(3-1-3-3)$ (3-1-3-3).

\section{Malgacheliodes guillaumeti n. gen., n. sp.}

MATÉRIEL TYPE. - Madagascar, Amindramiova, sur la rive gauche du cours supérieur du Zomandao, forêt dense humide de montagne, altitude $1950 \mathrm{~m}$, J.-M. Betsch coll., 18.I.1971, 12 adultes (6 o o $0^{7}, 6$ \% 9 ) syntypes (en alcool) (MNHN-Ac1149).

LOCALITÉ TYPE. - Madagascar, Amindramiova, sur la rive gauche du cours supérieur du Zomandao, entre 1850 et 1950 m, RCP 225, station de l'Andringitra, Mad. 282.

ÉTYMologie. - Cette espèce est dédiée au Professeur J.-L. Guillaumet, membre de l'équipe de la RCP 225, botaniste retraité du centre ORSTOM de Tananarive (voir Paulian et al. 1971, 1973).

DiAGNOSE. - Adultes: poil ro latéral, sous la visière. Poil in sur un promontoire. Sensillus en massue aplatie. Lyrifissures $i a, i m$, ip, ih, ips présentes; ip en forme de demi-cercle. Bordures épimériques ne correspondant pas exactement avec les apodèmes respectifs; demi-apodèmes 2 et $s j$ terminant loin du plan de symétrie. Entre les volets génitaux et anaux, large sillon partant en oblique. Fémurs trachéens, carénés; trachées I et II à ouverture dorsale, III et IV s'ouvrent dans la gorge dorsale; tibias II, III et IV avec crête, terminée en pointe; formule des pattes: I (1-5-2-4-18-3) (1-2-2); II (1-5-2-4-16-3) (1-1-2); III (2-3-3-4-15-3) (1-1-0) ; IV (1-3-2-4-12-3) (0-1-0). Extrémité des barbules des poils infracapitulaires pourvue d'un orifice.

Immatures: trachée I bien visible chez la deutonymphe, à peine visible chez la proto- et la tritonymphe. Crispins complets: bien développées sur tarses, tibias et génuals dès la larve; crispin du fémur IV bien visible à partir de la deutonymphe. $\varphi_{1}$ et $\varphi_{2}$ dans des cuvettes indépendantes dès la deutonymphe. Formules des pattes: larve et protonymphe: I (0-2-2-3-16-1); II (0-2-2-3-13-1); III (0-2-1-2-13-1); protonymphe: IV (0-0-0-0-7-1); deutonymphe: I (0-43-4-16-1); II (0-4-3-5-15-1); III (1-3-2-4-15-1); IV (1-2-2-4-12-1); tritonymphe: I (0-5-3-4-18-1); II (0-43-5-15-1); III (1-3-2-4-15-1); IV (1-2-3-4-12-1).

\section{DESCRIPTION DES ADULTES}

Animaux portant les scalps des stases précédentes.

\section{Taille, forme et couleur}

Taille: animaux mesurés au MEB : longueur de 510 à $630 \mu \mathrm{m}$ (moyenne sur 10 exemplaires, $545 \mu \mathrm{m}$ ); 

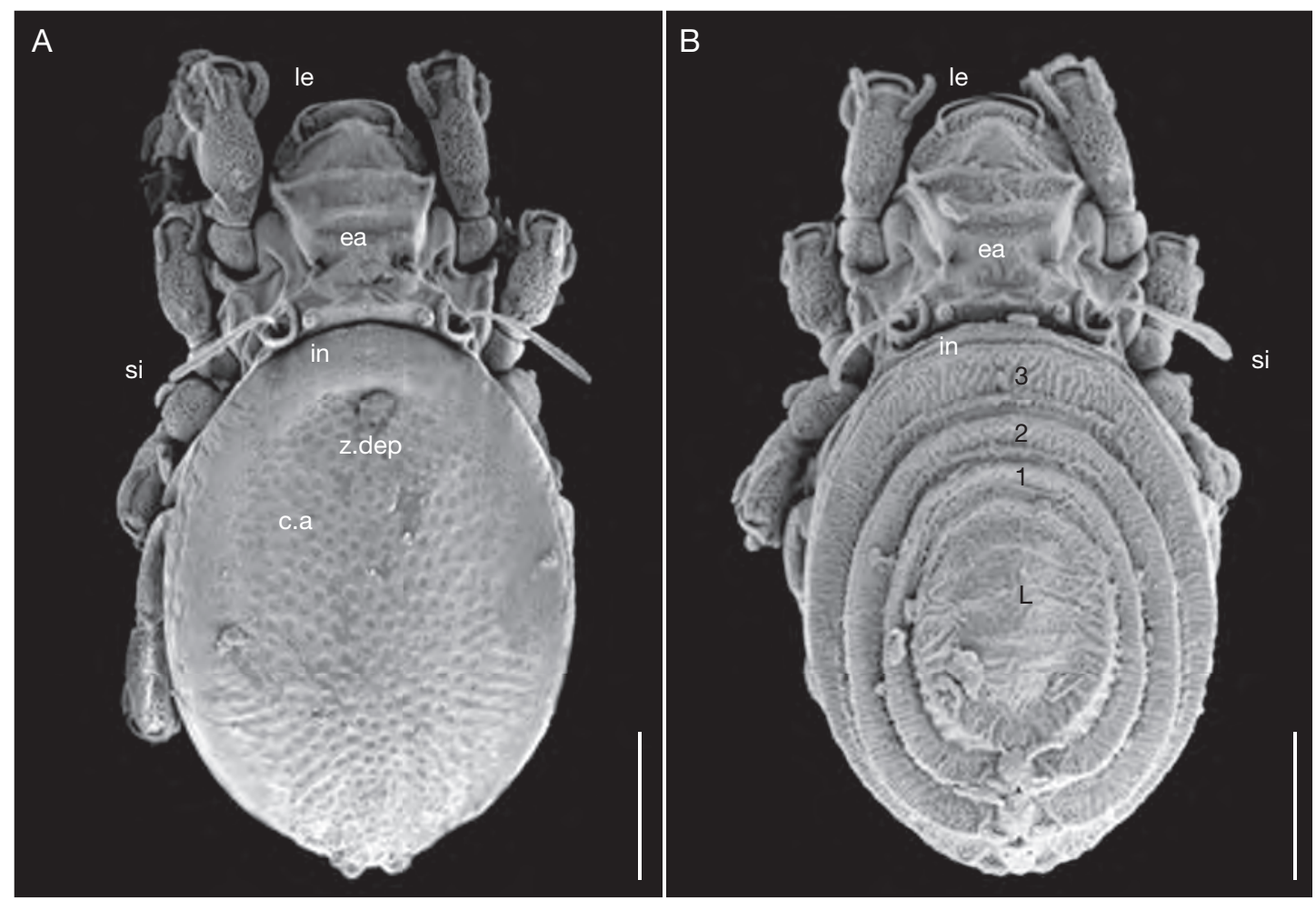

FIG. 1. - Malgacheliodes guillaumeti n. gen., n. sp., adulte, vue dorsale: A, scalps enlevés; B, avec les scalps des stases précédentes. Échelles: $100 \mu \mathrm{m}$. Abréviations: voir Matériel et méthode.

largeur de 270 à $320 \mu \mathrm{m}$ (moyenne $290 \mu \mathrm{m}$ ); animaux mesurés au microscope optique, dans l'acide lactique à froid et en lame creuse: longueur de 580 à $650 \mu \mathrm{m}$ (moyenne sur 10 exemplaires, $620 \mu \mathrm{m}$ ); largeur de 350 à $470 \mu \mathrm{m}$ (moyenne $415 \mu \mathrm{m})$.

Pas de différences de taille entre mâles et femelles.

Forme: animaux un peu allongés (Figs $1 \mathrm{~A} ; 2 \mathrm{~B}$; $5 \mathrm{~A})$, à peine convexes. Sex-ratio: normal (1:1).

Couleur (en lumière réfléchie): marron, terne et mat (animaux avec cérotégument); brun clair à brun foncé (animaux sans cérotégument).

\section{Cérotégument}

Présent partout; formé par une couche basale (c.b) (Fig. 3C) et des colonnes qui s'en élèvent (Fig. 3A-D).

Couche basale: épaisseur $0,5 \mu \mathrm{m}$, lisse; suit les inégalités de la cuticule; à l'intérieur des concavités arrondies de la cuticule du notogaster, présence de minces filaments (fi) (Fig. 3D).

Colonnes: constituées de petites boules (p.p) qui leur confèrent un aspect poreux; disposées en cercles de cinq ou six unités (Fig. 3B, C).

Elles sont de trois types: 1 ) grandes (c.g): coniques au bout arrondi (Fig. 3B); de 2,1 à 3,1 $\mu \mathrm{m}$ de long et de 0,7 à $1,4 \mu \mathrm{m}$ de large; partie basale et partie apicale de 0,5 à $0,9 \mu \mathrm{m}$ (Fig. 3B); distribuées sur le prodorsum et le bouclier ventral (Fig. $5 \mathrm{~F}$ ); 2) moyennes (c.m.l): cylindriques; longueur 1,5 à $2,0 \mu \mathrm{m}$, largueur 1,7 à $2,7 \mu \mathrm{m}$ (Fig. 3A, D); distribuées sur : la cuticule lisse, principalement sur le prodorsum et la partie dorsale du notogaster (elles sont moins nombreuses dans la zone cuticulaire avec microsculpture à concavités, cf. Fig. 3D); la paroi latérale; le bouclier ventral; 3 ) petites (c.p): longueur 0,3 à $1,4 \mu \mathrm{m}$, largueur 0,3 à $1,1 \mu \mathrm{m}$ (Fig. 3A); présentes sur: prodorsum, notogaster 


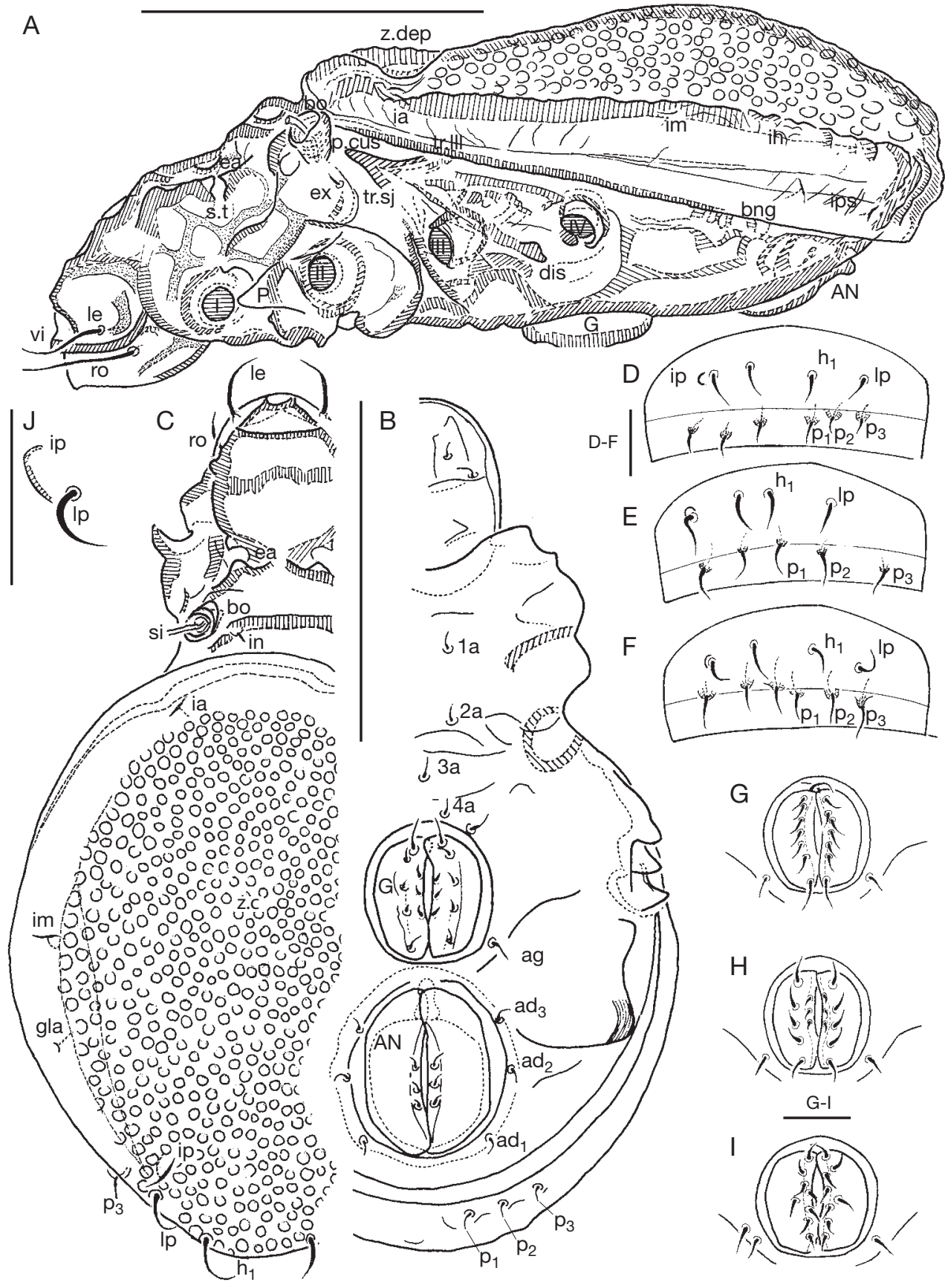

FIG. 2. - Malgacheliodes guillaumeti n. gen., n. sp., adulte: A, vue latérale; B, vue dorsale; C, vue ventrale; D-F, vue ventrale de la zone épimérique, schématisée; G-I, partie postérieure du notogaster; J, poil lp et lyrifissure ip. Échelles: A-C, $100 \mu \mathrm{m}$; D-F, $10 \mu \mathrm{m}$; G-I, $20 \mu \mathrm{m} ; \mathrm{J}, 10 \mu \mathrm{m}$. Abréviations: voir Matériel et méthode. 

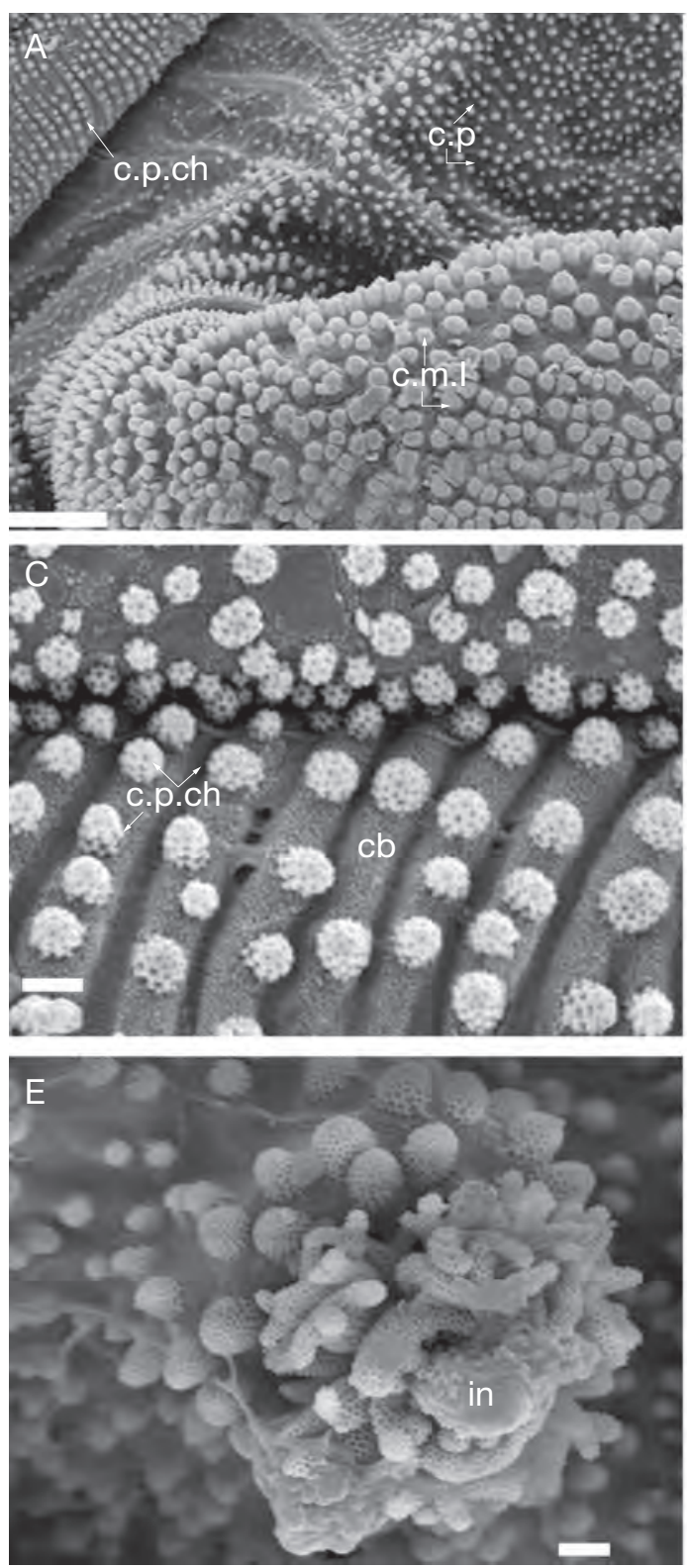
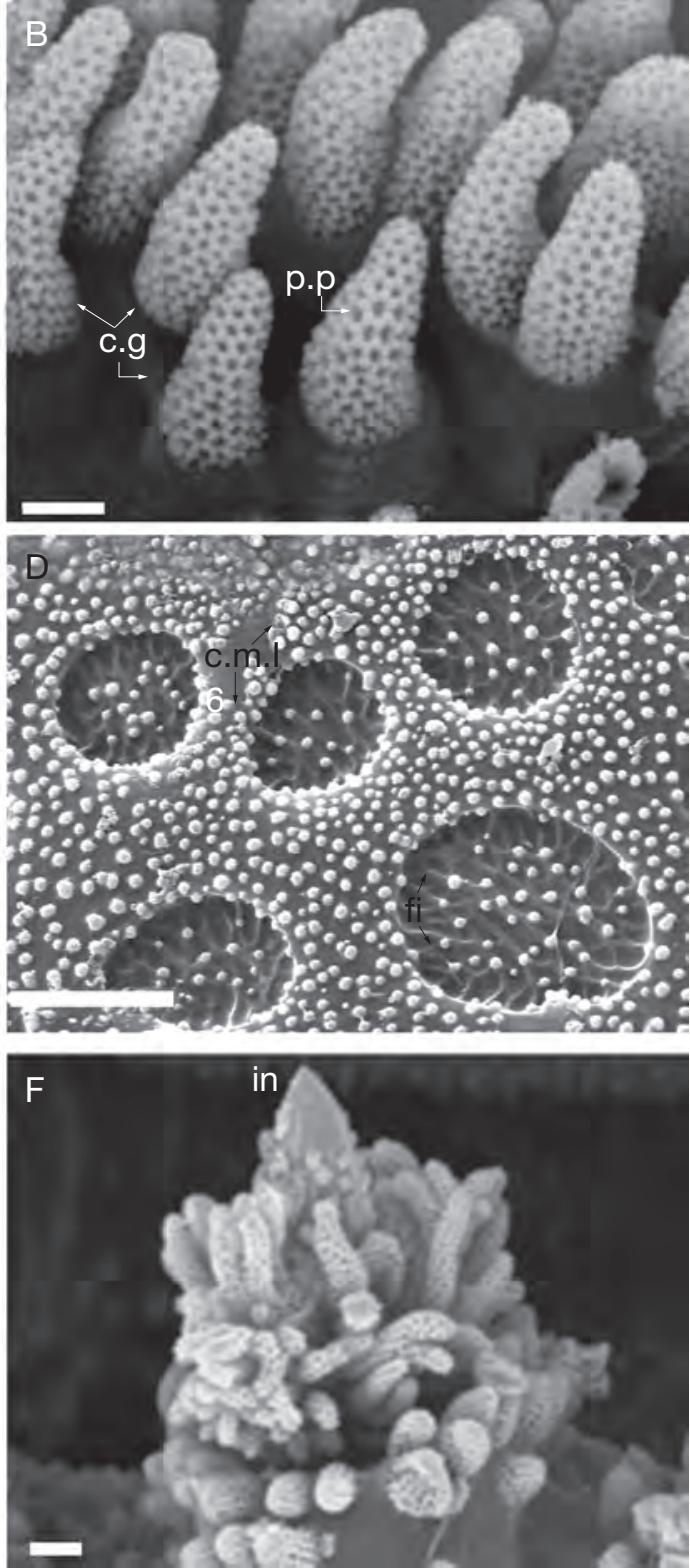

FIG. 3. - Malgacheliodes guillaumeti n. gen., n. sp., adulte, cérotégument: A, fémur de la patte IV, et, à l'arrière plan, le bord du notogaster et la partie latérale du corps; $\mathbf{B}$, détail du cérotégument, côté ventral, près de la ceinture séjugale; $\mathbf{C}$, détail du cérotégument, c.p.ch; D, détail du cérotégument, notogaster; E, poil in, vue dorsale; F, poil in, vue latérale. Échelles: A, D, $10 \mu \mathrm{m} ; \mathrm{B}, \mathrm{C}, \mathrm{E}, \mathrm{F}, 1 \mu \mathrm{m}$. Abréviations: voir Matériel et méthode.

(sauf sur les bords), bouclier ventral, podosoma, pattes et certains poils (principalement des pattes, Fig. 7D).
Outre les colonnes, le cérotégument présente également des structures ayant la forme de petits choux-fleur (c.p.ch) (Fig. 3A, C) d'environ 0,5 $\mu \mathrm{m}$, 

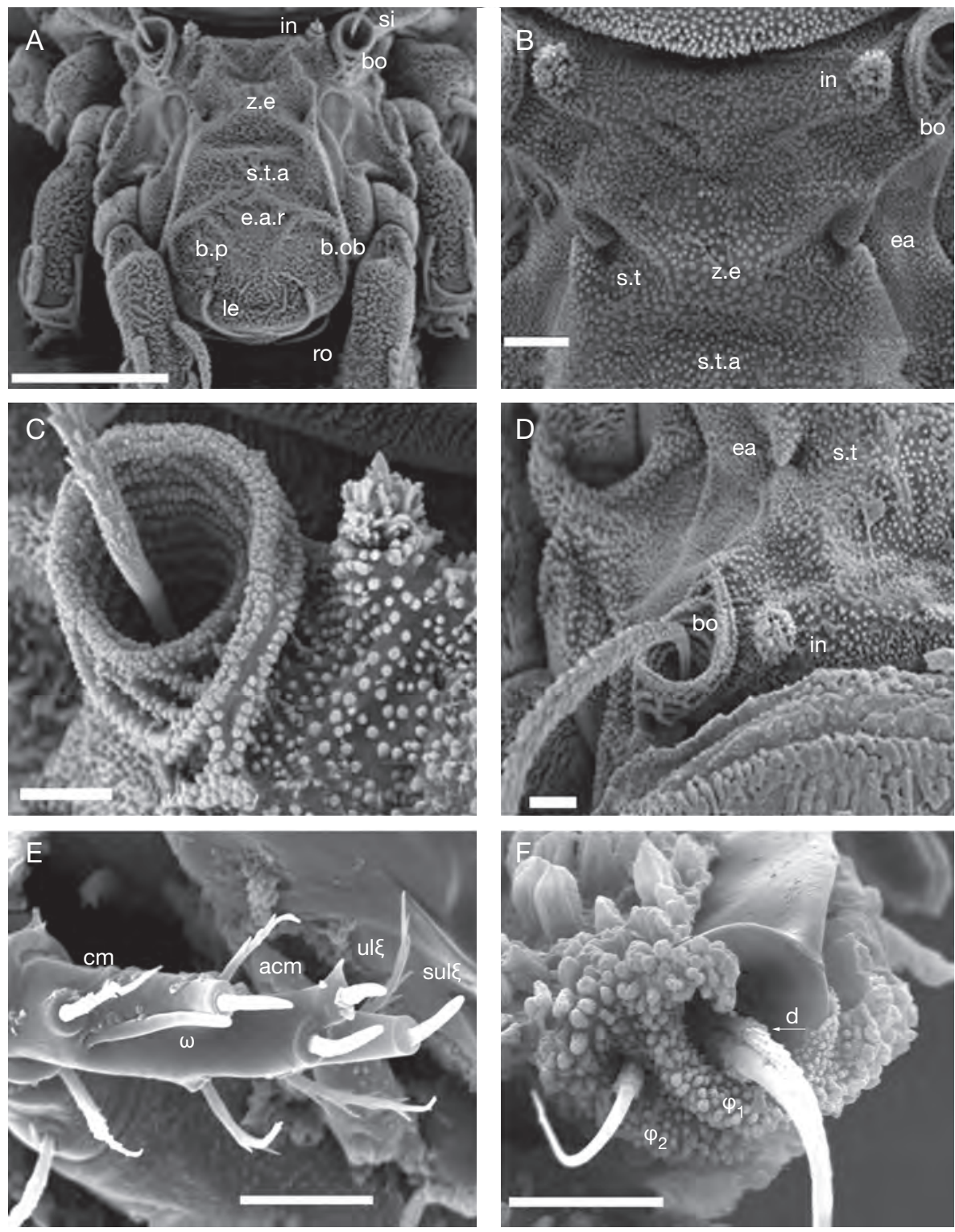

FIG. 4. - Malgacheliodes guillaumeti n. gen., n. sp., adulte: A, prodorsum, vue frontale; B, région des énanthiophyses, vue dorsale; $\mathbf{C}$, bothridie et poil in, vue frontale; D, bothridie et poil in, vue dorsale; E, palpe, vue dorsale. F, apophyse du tibia I, avec ses 2 cuvettes. Échelles: A, $100 \mu \mathrm{m}$; B-F, $10 \mu \mathrm{m}$. Abréviations: voir Matériel et méthode.

sur le prodorsum, et sur la paroi latérale du notogaster au niveau du tectum de bordure entre le bord libre bng et la ligne $\lambda$. Pattes recouvertes totalement par le cérotégument (en partie visible dans la Figure 5A).

\section{Cuticule}

Microsculpture de la cuticule complexe, se présentant sous différents aspects: des zones lisses, principalement sur les pattes (Fig. 4F); des zones avec des irrégularités (Figs 1A;2B), sur le prodorsum, sur 

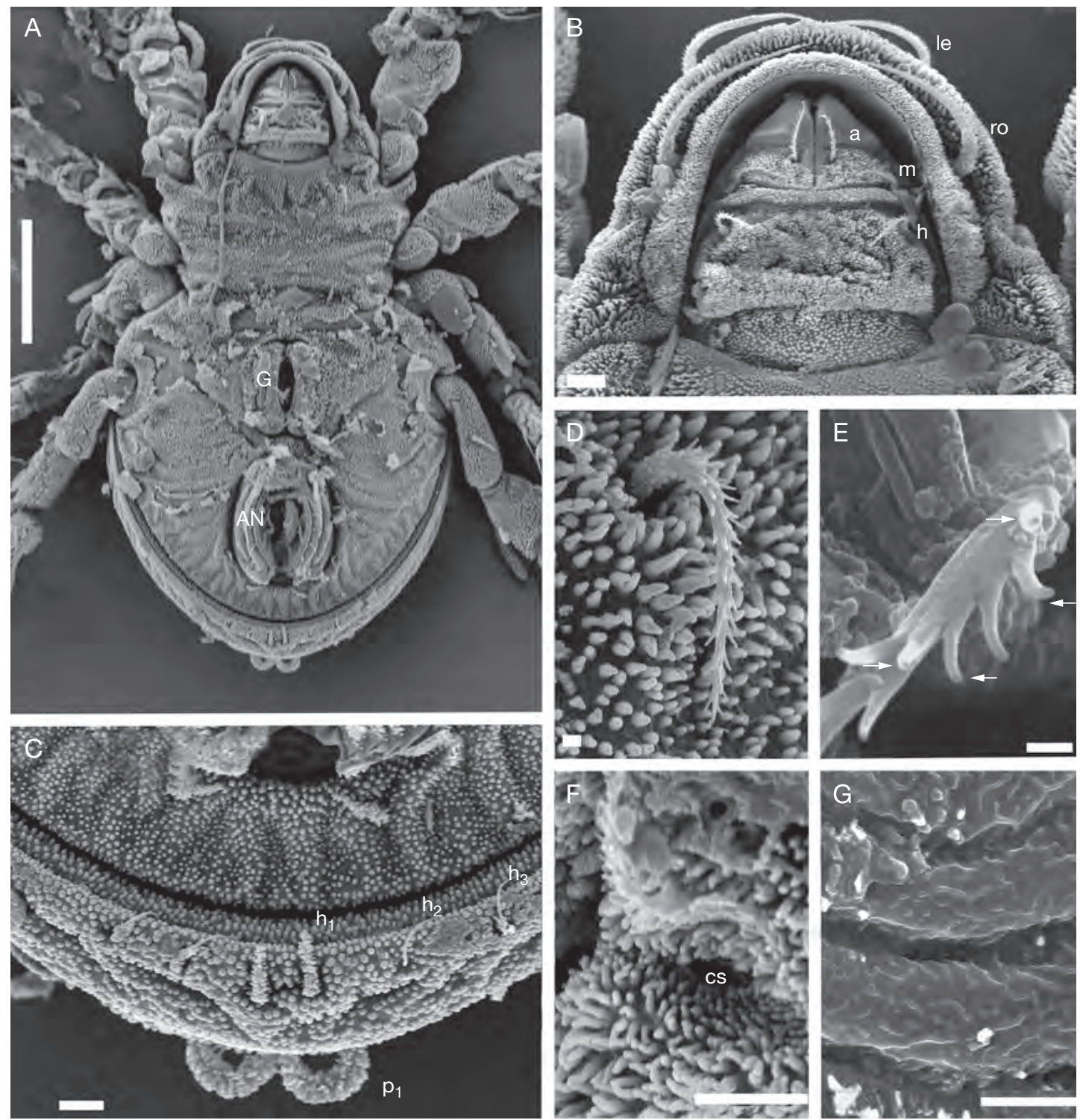

FIG. 5. - Malgacheliodes guillaumeti n. gen., n. sp., adulte: A, vue ventrale; B, infracapitulum; C, région postérieure; D, poil épimérique; $\mathbf{E}$, détail des barbules du poil $a$, les flèches indiquent les orifices situés au bout des barbules; $\mathbf{F}$, zone du sillon séjugal, ceinture; G, sillons parallèles en demi-cercle, partie postérieure de l'ouverture génitale. Échelles: $A, 100 \mu \mathrm{m} ; B, C, F, G, 10 \mu \mathrm{m} ; \mathrm{D}, \mathrm{E}, 1 \mu \mathrm{m}$. Abréviations: voir Matériel et méthode.

le notogaster en position dorsale antiaxiale et sur le bouclier ventral; des zones avec des microsculptures à concavités peu profondes, arrondies, délimitées par des bords nets, dans la partie centrale du notogaster
(Fig. 2A, C); des zones avec des sillons, sur la paroi latérale du notogaster (Fig. 2A); des zones avec des épaississements, sur le bouclier ventral, surtout en bordure (Fig. 6A); des petites bosses 
alignées (apparaissant sous forme de minces sillons en microsopie optique) (Fig. 6B) et des dépressions irrégulières, dans la zone des épimères (Fig. 6C).

\section{Chaetotaxie}

Poils du corps sans le cérotégument: ils sont de trois types: 1) lisse à pointe éfilée: poils notogastraux, génitaux et anaux (Fig. 2A-C, D-F, G-I respectivement) ; 2) lisse, court: poil in (Fig. 1C); et 3) barbelé: ro (Fig. 7F), épimériques (Fig. 7B), infracapitulaires (Fig. 5D); les barbules des poils infracapitulaires présentent un orifice à leur extrémité (Fig. 5E). Le cérotégument confère aux poils un aspect particulier: poil in, voir Figures 3E, F; 4C; pour les autres poils voir les Figures $5 \mathrm{C}$ et $7 \mathrm{D}$.

La chaetotaxie des pattes est traitée au paragraphe Pattes.

\section{Scalps}

Toujours présents. Pas de réticulations posthumes ni de substance adhésive.

\section{Prodorsum}

Forme: cf. Figs 1A, B ; 2A, C. Enantiophyses (e.a) présentes; le sillon transversal (s.t) passe entre elles, ne traverse pas le plan de symétrie et s'arrête tout près des e.a (Figs $2 \mathrm{~A}, \mathrm{C} ; 4 \mathrm{~A}, \mathrm{~B}, \mathrm{D})$.

Zone paraxiale élevée (z.e) au niveau des e.a; vers l'avant, sillon transversal antérieur (s.t.a) traversant le plan de symétrie (Fig. 4A, B) ; s.t.a limité postérieurement par z.e, latéralement par deux épaississements cuticulaires (e.c.o) et antérieurement par des épaississements ramifiés et complexes (e.a.r, épaississements antérieurs ramifiés).

e.a.r comporte deux branches parallèles (b.p) dirigées vers le capuchon rostral et deux autres branches obliques $(b . o b)$ qui se rejoignent près $\mathrm{du}$ plan de symétrie; des $b . o b$ se détachent une série d'épaississements obliques vers le plan de symétrie (Fig. 4A ). Capuchon rostral lisse (Fig. 2A ).

Bothridie de forme particulière, proéminente, en large cornet (Figs 1A, B; 2A, C; 4 A, C, D). En microscopie optique l'observation de la vue latérale est difficile, les anneaux externes et internes se chevauchant; la meilleure observation est en vue dorsale légèrement inclinée vers l'avant (Fig. 2C) : ouverture dorsale inclinée vers l'avant (Fig. 4A, C,
D); rebord postérieur plus haut que l'antérieur; anneaux internes plus au moins circulaires (Fig. 4A, C); anneau le plus externe ovoïde avec un angle antérieur qui lui confere sa forme caractéristique (Fig. 4C); partie la plus interne du cornet se dirigeant de l'arrière vers l'avant.

Poil in recouvert de cérotégument (sauf sa partie apicale) (Fig. 3E, F) lui donnant sa forme spécifique; taille environ $10 \mu \mathrm{m}$, terminé en pointe (Fig. 3F), inséré sur un promontoire (Fig. 4C). Sensillus grand, en massue flabelliforme aplatie et spatulée (Fig. 7E) parsemée de petites barbules: la tige s'amincit fortement à partir du niveau de l'ouverture de la bothridie vers l'intérieur; barbules beaucoup moins nombreuses à proximité de l'ouverture bothridiale. Poil ex placé latéralement, sous la bothridie (Fig. 2A).

Capuchon rostral avec une visière latérale $(v i)$ (Fig. 2A). Poils le et ro barbelés (Fig. 7F) extrémité des barbules semblant trouée; le placé au dessus de $v i$, inséré sur promontoire éloigné du bord rostral (Fig. 2A); ro inséré au dessous de $v i$, bien en arrière de le et à la hauteur du poil infracapitulaire $a$ (Figs 2A; 5B).

\section{Notogaster}

Sans dorsophragmas ni pleurophragmas. Sillon dorsoséjugal large et profond (Figs 1A, B; 4B), assez plat. Dans sa partie antérieure une zone déprimée (z.dep) ; zone centrale (z.c) faiblement bombée, avec microsculpture faite de concavités arrondies (c.a), entourée d'une zone sans $c . a$ (Fig. 2C). Dépression circummarginale peu profonde (Figs 1A;2A); carène de bordure peu élevée et assez aplatie. Partie latérale du notogaster verticale (Fig. 2A).

À l'arrière et latéralement, tectum important s'étendant entre bng et $\lambda$ (Fig. 2A).

Chaetotaxie: poils en nombre variable (Fig. 2B, D-F), le plus souvent 5 paires $\left(h_{1}, l p, p_{1}, p_{2}, p_{3}\right)$; poils $p$ distribués de façon un peu aléatoire (Figs 2B, D-F; 5C).

Cinq paires de lyrifissures: $i a$, im, ip, placées dorsalement, ip près du poil $h_{1}$ et en forme de demi-cercle (Fig. 2E, F, G, K) ; ih et ips latérales (Fig. 2A).

Gla dépassant im vers l'avant et s'étendant jusqu'à ip vers l'arrière (Fig. 2C); débouché situé entre im et $i p$. 


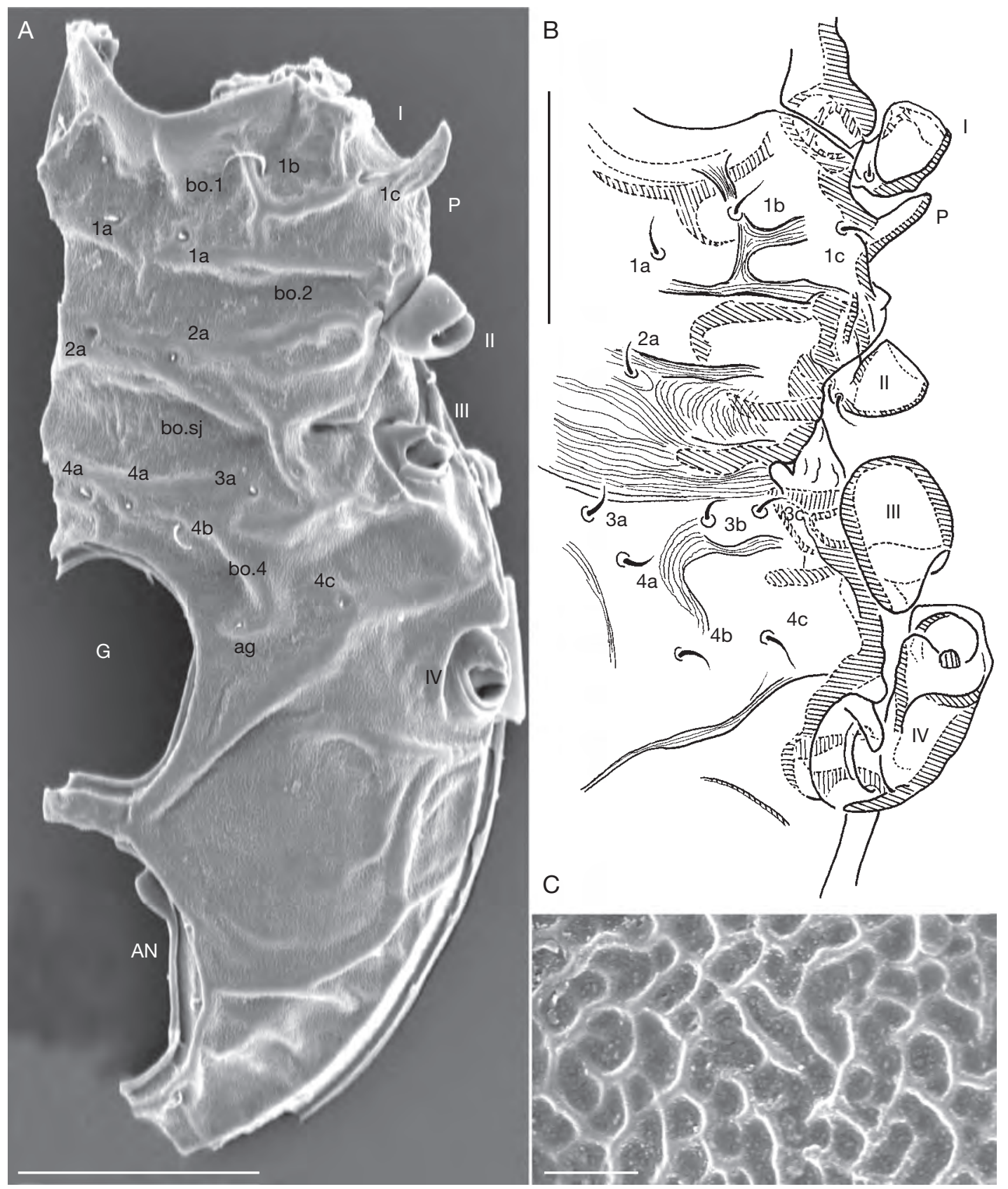

FIG. 6. - Malgacheliodes guillaumeti n. gen., n. sp., adulte: A, vue ventrale, cérotegument enlevé; B, idem, dessin d'après une

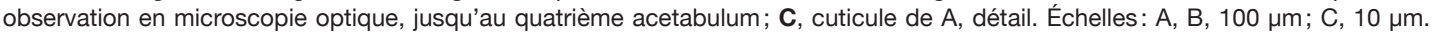
Abréviations: voir Matériel et méthode. 

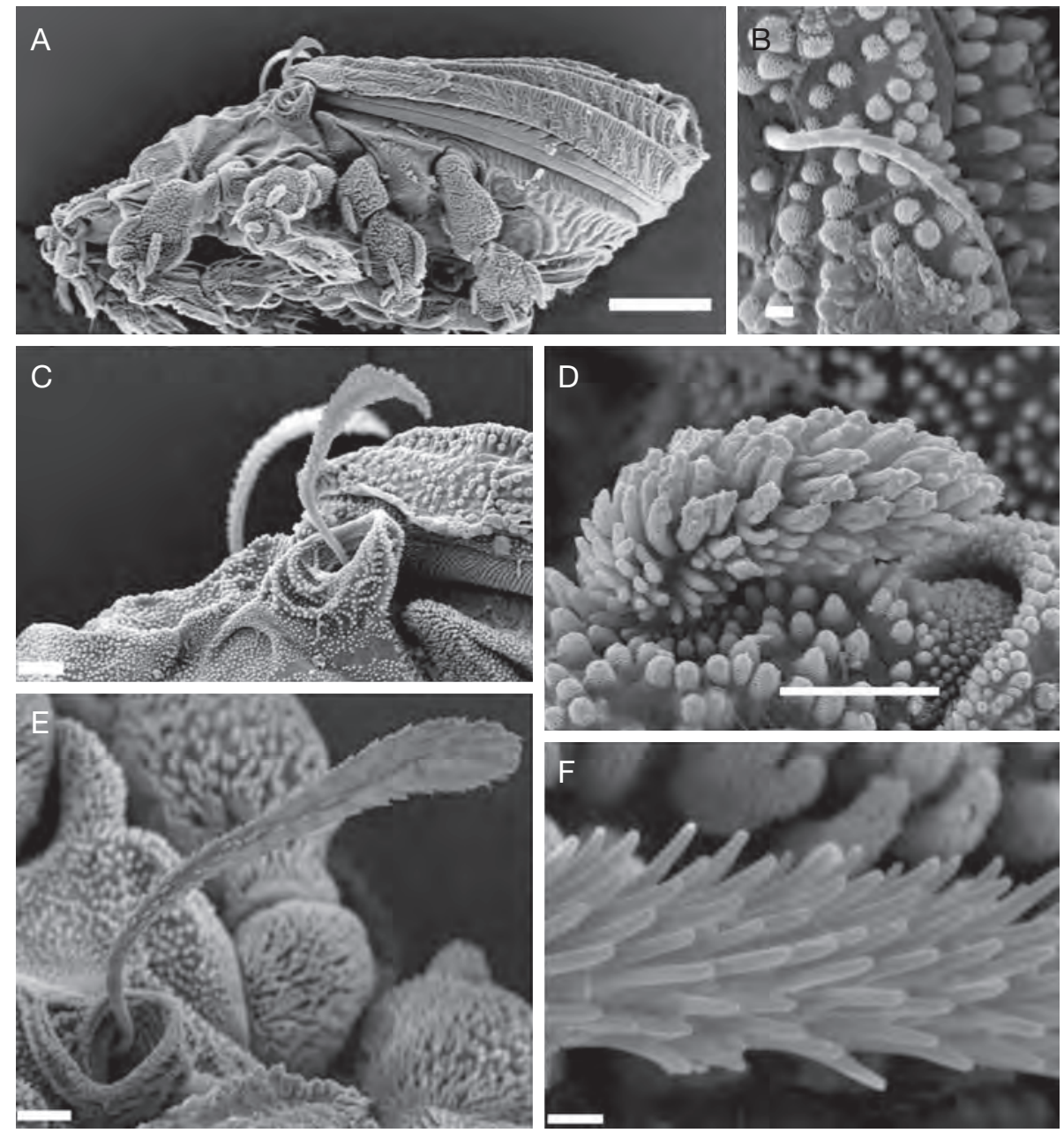

FIG. 7. - Malgacheliodes guillaumeti, n. gen., n. sp., adulte: A, vue latérale générale; B, poil épimérique 1b; C, bothridie et sensillus, vue latérale; D, poil des pattes, enrobé du cérotégument; E, sensillus vue latérale; F, poil ro, barbules. Échelles: A, $100 \mu \mathrm{m}$; B, F, $1 \mu \mathrm{m} ; \mathrm{C}-\mathrm{E}, 10 \mu \mathrm{m}$. Abréviations: voir Matériel et méthode.

\section{Caractères ventraux}

Région épimérique particulière (Figs $2 \mathrm{~B} ; 5 \mathrm{~A}, \mathrm{~B}$; $6 \mathrm{~A}, \mathrm{~B})$, les bordures épimériques (bo) ne contenant pas toujours la base de l'apodème correspondant; présence de sillons et d'épaississements transversaux sur les épimères (Figs 2B; 6A, B).

Apodèmes (cf. Figs 2B et 6B); 2 et sj sont des demiapodèmes terminant loin du plan de symétrie.

Chaetotaxie épimérique (3-1-3-3); parfois une faible néotrichie (symétrique et asymétrique) au niveau du quatrième épimère, niveau $4 a, 4 b$. Poils de grande taille (Figs 2C; 7B). Sillon séjugal large et profond; existence d'une véritable ceinture antiaxiale en forme de $U$, avec énantiophyse antérieure, au fond de laquelle se trouve le vestibule séjugal où loge la trachée séjugale (Figs $5 \mathrm{~A}, \mathrm{~B}$, $\mathrm{F} ; 6 \mathrm{~B})$.

Des sillons parallèles en arc de cercle s'observent au niveau de la région antérieure de l'ouverture anale (Figs 5E; 6A) ainsi que des courts sillons rayonnant depuis le rebord du bouclier ventral (Fig. 5A, C). 


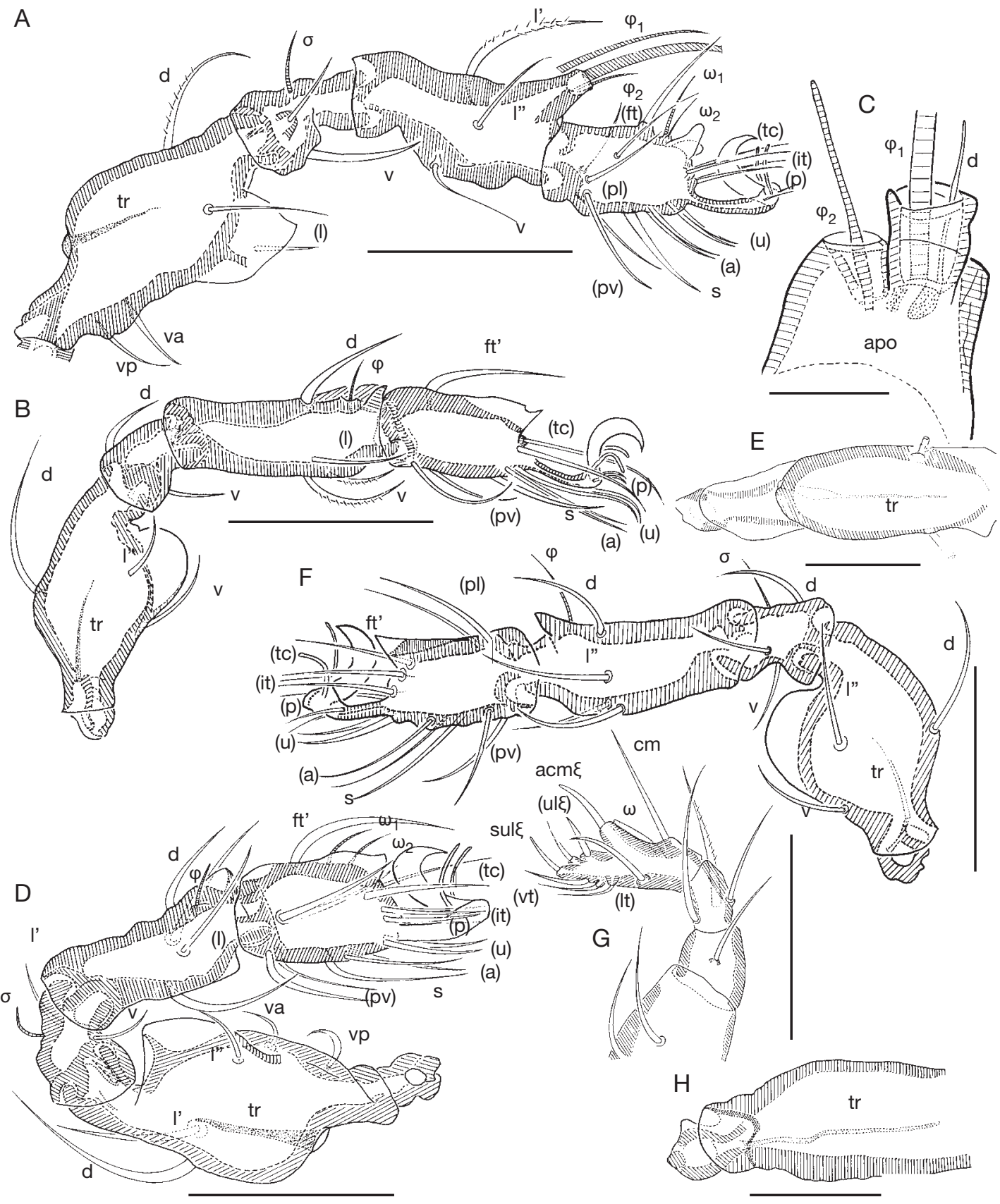

FIG. 8. - Malgacheliodes guillaumeti n. gen., n. sp., adulte: A, patte I, vue antiaxiale; B, patte III, vue paraxiale; C, tibia I, apophyse antilatérodorsale avec ses cuvettes; $\mathbf{D}$, patte II, vue antiaxiale; E, fémur II, vue dorsale simplifiée montrant la trachée; $\mathbf{F}$, patte IV, vue paraxiale; G, palpe, vue antiaxiale; H, fémur IV, vue dorsale simplifiée pour montrer la trachée. Échelles: A, B, D, 50 m; C, H, $20 \mu \mathrm{m}$; $\mathrm{E}, \mathrm{G}, 25 \mu \mathrm{m} ; \mathrm{F}, 70 \mu \mathrm{m}$. Abréviations: voir Matériel et méthode. 
Entre les volets génitaux et anaux, un large sillon oblique s'étend jusqu'aux environs du quatrième acetabulum, où l'on observe un plateau plus ou moins carré avec éperon. À l'arrière de ce plateau se trouve une série de sillons et d'épaississements (Figs 2B; 6A, B).

Chaetotaxie génitale: $\mathrm{G}$ : 8 ; distribution la plus fréquente en deux rangées, 5 antiaxiaux et 3 paraxiaux (Fig. 2B, H, I); un seul cas observé où les poils sont distribués pratiquement sur une seule rangée (Fig. 2G); tailles variables (Fig. 2B, G-I); faible néotrichie en général asymétrique (Fig. 2I); une paire de poils ag. Lyrifissures: ian, en forme de pore; iad semble absente.

\section{Système trachéen}

Trachées I, sj et III présentes; I en position habituelle, structure très mince, très régressive; $s j$ et III normales.

\section{Caractères latéraux}

Présence de nombreuses carènes fragmentant la structure générale (Fig. 2A).

Apophyse pédotectale (P) (Fig. 2A). Discidium (dis) important, à partie distale arrondie (Fig. 2A). Importante pointe custodiale (p.cus) partant près du bord du bouclier ventral et finissant juste en arrière de la bothridie, dirigée de l'arrière vers l'avant, terminée en pointe (Fig. 2A). Sillon séjugal profond, oblique ou l'on peut voir le vestibule et tr.sj (Fig. 2A).

Disposition des acetabula: I-III en position normale, IV placé assez haut, à la limite du bouclier ventral (Fig. 2A). Trous acétabulaires I, II, III à ouverture nettement latérale, celle de IV beaucoup plus ventrale (Figs 2C; 7A).

\section{Gnathosoma}

Infracapitulum diarthre à grand menton; poils $a, m, h$ barbelés; barbules avec un orifice apical (Fig. 5E).

Palpe (Figs 4E; 8G) de formule (0-2-1-3-9), plus solénidion $\omega$ placé paraxialement, éloigné de acm $\zeta$; eupathidies sul $\zeta,(u l) \zeta$ et acm $\zeta$ de forme particulière (Fig. 8G).

\section{Pattes}

Tridactyles hétérodactyles; ongle central fort, épais, coloré, ongles latéraux grêles, peu colorés; entiè- rement recouvertes par le cérotégument; cuticule lisse; la patte IV est la plus grande, puis I, III , II (Fig. 8A, B, D, F).

Crispins complets sur génuals, tibias et tarses; simples, arrondis, plus développés ventralement que dorsalement; crispins partiels sur les fémurs, développés dorsalement, absents ventralement, en position paraxiale pour les fémurs I et II et antiaxiale pour III et IV.

Tarses: derrière la griffe, en falaise, pente de la falaise en angle aigu; tarses II, III et IV avec une grande carène lamelliforme dorsale terminée en pointe (Fig. 8B-D); carène moins importante, divisée en deux, sur le tarse I (Fig. 8A); style long sur I, II et III, très long sur IV; les poil proraux $(p)$ sont implantés dorsalement près de leur extrémité.

Tibias II, III et IV avec crête terminée en pointe; celle du tibia I se termine en une importante apophyse antidorsolatérale avec deux cuvettes : la plus grande, située du côté antiaxial apophysaire, loge le solénidion $\varphi_{1}$ et le petit poil compagnon $d$ collé au solénidion; la plus petite, en position paraxiale, loge le solénidion $\varphi_{2}$ (Figs 4F; 8A-D, F).

Fémurs: trachéens, à grande carène lamelliforme ventrale; trachées longues, à extrémité arrondie (Fig. 8A, B, D-F, H); celles des fémurs I et II différentes de celles des fémurs III et IV: I et II (Fig. 8E, $\mathrm{H})$ avec un gros stigmate, qui s'ouvre librement en surface à l'arrière, dorsalement; parcours trachée particulier: large près du stigmate puis aminci régulièrement; son extrémité arrondie (Fig. $8 \mathrm{E}$ ); trachées III et IV à stigmate petit, difficile à voir, caché par le crispin et s'ouvrant dans la gorge dorsale (Fig. $8 \mathrm{H}$ ).

Formules des poils et solénidions: I (1-5-24-18-3) (1-2-2); II (1-5-2-4-16-3) (1-1-2); III (2-3-3-4-15-3) (1-1-0); IV (1-3-2-4-12-3) $(0-1-0)$.

\section{IMMATURES}

Taille, forme, couleur

Larve: $250-290 \mu \mathrm{m}(280 \mu \mathrm{m})$; protonymphe: 360-393 $\mu \mathrm{m}(376 \mu \mathrm{m})$; deutonymphe: 430$451 \mu \mathrm{m}(421 \mu \mathrm{m})$; tritonymphe: $520-560 \mu \mathrm{m}$ $(513 \mu \mathrm{m})$. 


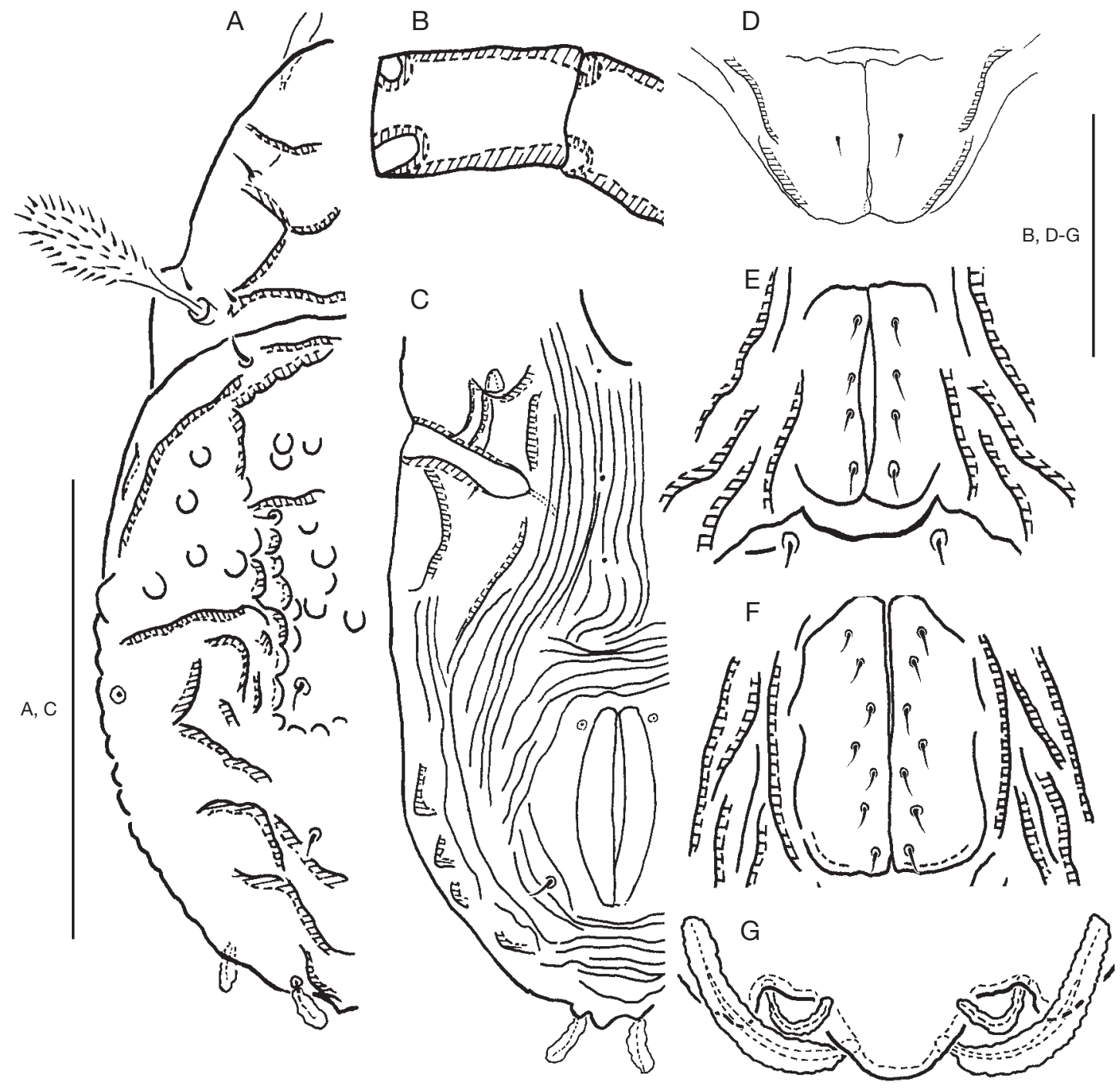

FIG. 9. - Malgacheliodes guillaumeti n. gen., n. sp., stases immatures: A, larve, vue dorsale partielle; B, larve, genual et tibia I; $\mathbf{C}$, larve, vue ventrale partielle; $\mathbf{D}$, protonymphe, région génitale; $\mathbf{E}$, deutonymphe, région génitale; $\mathbf{F}$, tritonymphe, région génitale; G, trinonymphe, croupion. Échelles: A, C, $100 \mu \mathrm{m} ; \mathrm{B}, \mathrm{D}-\mathrm{G}, 30 \mu \mathrm{m}$. Abréviations: voir Matériel et méthode.

Toutes les stases sont assez aplaties. Les larves sont blanchâtres, mates, ternes; les nymphes à peine jaune clair: seule les boucliers (prodorsal, pygidial, du menton, des joues) et les articles des pattes sont un peu plus colorés, marron très clair.

Cérotégument du même type que chez l'adulte; moins développé chez la larve, plus développé chez la tritonymphe.

\section{Cuticule}

Larve: prodorsum avec sillons et épaississements cuticulaires; région gastronotique plissée avec quelques dépressions ovoïdes arrondies (Fig. 9A); ventralement, forte et largement plissée (Fig. 9C). Nymphes: prodorsum voisin de celui de l'adulte; région gastronotique plissée ; concavités ovoïdes arrondies bien visibles, plus marquées de la 
protonymphe à la tritonymphe, rappellant celles de Pherolidoles wehnckei (Grandjean 1964: figs 6, 7). Ventralement la cuticule est fortement plissée chez toutes les stases.

\section{Poils}

Petits à pointe aiguë, enrobés par le cérotégument.

\section{Prodorsum}

Pas de différence notable entre la larve et les nymphes (Fig. 9). Dès la stase larvaire, le prodorsum ressemble à celui de l'adulte; capuchon rostral échancré; bothridie et sensillus plus petits mais semblables à ceux de l'adulte; poils lamellaires plus petits que les rostraux.

\section{Région gastronotique}

Celle de la larve très différente de celle des nymphes.

Larve (Fig. 9A, C) : faiblement bombée vers l'arrière et aplatie vers l'avant; concavités arrondies et plis délimitent une zone moyenne qui atteint le niveau du poil $d m$; à l'arrière du poil $d p$ une série de plis qui, atteignant la zone paraxiale, se courbent et se dirigent vers l'arrière.

Nymphes: croupion présent (Fig. 9G); zone d'insertion des poils $p_{1}$ arrondie; $p_{1}$ grand, $h_{1}$ petit, recouverts de cérotégument.

Formule gastronotique (7-6-5).

\section{Région ventrale}

Fortement plissée dès la stase larvaire (Fig. 9C); formule génitale: (1-4-7-8); aggénitale (0-1-1-1); coxisternales (3-1-2) (3-1-2-1) (3-1-3-2) (3-1-3-3) (3-1-3-3).

\section{Système trachéen}

Trachée I à peine visible chez la protonymphe et la tritonymphe, bien visible chez la deutonymphe; trachée $s j$ bien visible dès la larve; trachée III bien visible chez deuto- et tritonymphe.

\section{Pattes}

Crispins complets, bien développés du côté ventral et dorsal sur les tarses, tibias et genuals dès la larve (Fig. 9B); crispin dorsal sur le fémur IV, rudimentaire chez la protonymphe et bien visible chez la deutonymphe $\varphi_{1}$, dès la stase larvaire dans une cuvette; $\varphi_{2}$ apparaît chez la deutonymphe, placé en dehors de la cuvette qui contient $\varphi_{1}$; saccules des fémurs bien visibles dès la larve; tarses avec crêtes bien visibles à partir de la deutonymphe et styles longs dès la larve.

Larve et protonymphe: I (0-2-2-3-16-1); II (0-22-3-13-1) ; III (0-2-1-2-13-1); protonymphe IV (0-0-0-0-7-1); deutonymphe: I (0-4-3-4-16-1); II (0-4-3-5-15-1); III (1-3-2-4-15-1); IV (1-2-2-412-1); tritonymphe: I (0-5-3-4-18-1); II (0-4-3-515-1); III (1-3-2-4-15-1); IV (1-2-3-4-12-1).

\section{REMARQUES}

L'existence du poil compagnon $d$ sur le tibia I est intéressante à noter, car il est absent chez Pheroliodes wehnckei; Grandjean (1964) indique cependant avoir trouvé un spécimen pourvu du petit poil compagnon, d'un seul côté. Chez Malgacheliodes, le poil compagnon $d$ du tibia I est toujours présent.

L'existence d'un orifice à l'extrémité des barbules (dans cette note, poils ro, le et infracapitulaires) est probablement assez courante chez les oribates; nous l'avons également observé sur les barbules des poils $a, s, p v$ et $v$ des pattes de certaines espèces du genre Galumna von Heyden, 1826.

\section{Clé des genres de Pheroliodidae Paschoal, 1987}

1. Adultes sans les scalps des stases précédentes; notogaster avec ou sans concavités arrondies; volets génitaux carrés à rectangulaires

- Adultes portant les scalps des stases précédentes; cuticule du notogaster à concavités arrondies; volets génitaux arrondis

2. Cuticule du notogaster lisse; $\mathrm{G}: 8$ Nooliodes

- Cuticule du notogaster à concavités arrondies; G: 7 4 
3. Prodorsum normal; capuchon rostral sans visière; G: 7, en un seul rang; dent pédotectale présente; trachée I absente; fémurs non carénés et tibias sans crêtes; apophyse antilatérodorsale du tibia I sans cuvettes logeant les solénidions; pas de poil compagnon $d$........

- Prodorsum très complexe; capuchon rostral avec visière; G: 8 , en deux rangs; dent pédotectale, pointe custodiale et discidium présents; trachée I vestigiale; fémurs carénés; tibias avec crêtes; apophyse antilatérodorsale du tibia I avec deux cuvettes logeant $\varphi_{1}$ et $\varphi_{2}$; poil compagnon $d$ présent Malgacheliodes $\mathrm{n}$. gen.

4. Sensillus spatulé; notogaster avec sillon longitudinal entre les poils $h$; tarses tridactyles, ongles de taille moyenne ......................................................................... Lopholiodes

- Sensillus en massue ; notogaster sans sillon entre les poils $h$; tarses tridactyles, gros ongles denticulés Lyriffissella

\section{RÉFÉRENCES}

BALOGH J. 1962. - New Oribatids from Madagascar (Acari). Annales Historico-Naturales Musei Nationalis Hungarici 54: 419-427.

BAlogh J. \& BAlogh P. 1992. - The Oribatid Mites Genera of the World. Hungarian Natural History Museum, Budapest, Vol. 1, 263 p. et Vol. 2, 375 p.

Fernandez N. \& Cleva R. 2010. - Une nouvelle espèce de Scapheremaeus (Arachnida, Acari, Oribatida, Cymbaeremaeidae) de Madagascar: Scapheremaeus pauliani n. sp. Zoosytema 32 (1): 101-115.

GrandjEan F. 1964. - Pheroliodes wehnckei (Willmann) (Oribates). Acarologia 4: 353-386.

MAHUNKA S. 1997. — Oribatids of Madagascar III (Acari: Oribatida). (Acarologia Genavensia LXXXXIII). Revue suisse de Zoologie 104 (1): 115-170.

PAULIAN R. 1961. — La zoogéographie de Madagascar et des îles voisines. Faune de Madagascar, XIII. IRSM, Tananarive, $484 \mathrm{p}$.

Paulian R., Betsh J.-M., Guillaumet J.-L., BlanC C. \& Griveaud P. 1971. — Études des écosystèmes montagnards dans la région malgache. I. Le massif de l'Andringitra. 1970-1971. Géomorphologie, climatologie et groupements végétaux. Bulletin de la Société d'Ecologie 2 (2-3): 189-266.

Paulian R., Blanc C., Guillaumet J.-L., Betsh J.-M.,
Griveaud P. \& Peyrieras A. 1973. - Étude des écosystèmes montagnards dans la région malgache. II. Les chaînes Anosyennes. Géomorphologie, climatologie et groupements végétaux. (Campagne RCP 225, 1971-1972). Bulletin du Muséum national d'Histoire naturelle, sér. 3, 118: 1-40.

PASCHOAL A. 1987a. - A revision of the Plateremaidae (Acari: Oribatei). Revista Brasileira de Zoologia 3 (6): 327-356.

PASCHOAL A. 1987b. - A revision of the Pheroliodidae fam. n. (Acari: Oribatei). Revista Brasileira de Zoologia 3 (6): 357-384.

PASCHOAL A. 1989a. - Description of Nooliodes gen. n. and Nooliodidae fam. n. (Acari, Oribatei) from Madagascar. Revista Brasileira de Zoología 6 (2): 179-182.

PaschOAL A. 1989b. - Description of Lyrifissella gen. n. and Lyrifissellidae fam. n. (Acari, Oribatei). Revista Brasileira de Zoología 6 (2): 183-190.

Subias L. 2004 (baSE DE DONNÉES, ACTUALISÉE EN 2009). - Listado sistemático, sinonímico y biogeográfico de los Ácaros Oribatidos (Acariformes: Oribatida) del Mundo (excepto fósiles). Graellsia 60: 3-305. (http://www.ucm.es/info/zoo/Artropodos/ Catalogo.pdf).

Travé J. \& VACHON M. 1975. — François Grandjean 1882-1975 (notice biographique et bibliographique). Acarologia 17(1): 1-19. 